\title{
A CERTAIN CLASS OF INCIDENCE MATRICES
}

\author{
D. H. GOTTLIEB
}

1. Let $S$ be a set consisting of $K$ elements, and call any subset of $S$ containing precisely $m$ elements an $m$-set [2]. We wish to study incidence matrices obtained in the following manner: Let $K \geqq m \geqq n$ $\geqq 0$, we label the rows of the matrix by all the $m$-sets of $S$ and the columns by all the $n$-sets of $S$; take the $(i, j)$ element as 1 if the $m$-set corresponding to the $i$ th row contains the $n$-set corresponding to the $j$ th column, and zero otherwise.

This note studies the question of which collections of $m$-sets give rise to a linearly dependent set of row vectors, and likewise what combinations of $n$-sets give rise to a linearly dependent set of column vectors. To do this, we characterize the row null spaces and the column null spaces of the above matrices in an inductive manner. Then using this characterization, we prove that the above matrices must have maximal rank. A corollary then gives a necessary condition for the existence of a tactical configuration [1].

2. Now for any $K \geqq m \geqq n \geqq 0$, we shall define a canonical matrix $A_{m, n}^{K}$.

We first define a lexicographical ordering of the $m$-sets of $\{1, \cdots, K\}$, the set of in tegers from one to $K$, in the following way. Represent any $m$-set as $\left(a_{1}, \cdots, a_{m}\right)$ where $a_{i}<a_{i+1}$ for $i=1, \cdots, m$. Then we say that $\left(a_{1}, \cdots, a_{m}\right)<\left(b_{1}, \cdots, b_{m}\right)$ if and only if $a_{i}<b_{i}$ for the smallest value of $i$ for which $a_{i} \neq b_{i}$. We shall call this ordering the canonical ordering of the $m$-sets of $\{1, \cdots, K\}$.

We now define $A_{m, n}^{K}$ to be the incidence matrix whose rows correspond to the $m$-sets of $\{1, \cdots, K\}$ in their canonical order, and whose columns correspond to the $n$-sets in their canonical order. For example, the first row of $A_{m, n}^{R}$ corresponds to $(1,2, \cdots, m)$ and the first column to $(1,2, \cdots, n)$.

3. We gather some simple facts about $A_{m, n}^{K}$ here. $C_{a, b}$ will represent the usual binomial coefficient.

Lemma 1. $A_{m, n}^{K}$ has

(a) $C_{K, m}=K ! / m !(K-m) !$ rows,

(b) $C_{K, n}$ columns,

(c) $C_{m, n}$ ones in each row,

(d) $C_{K-n, m-n}$ ones in each column.

Received by the editors April 15, 1966. 
LEMMA 2.

(a) $A_{1,1}^{1}=A_{1,0}^{1}=A_{0,0}^{1}=A_{0,0}^{0}=1$,

(b) $A_{m, m}^{\boldsymbol{k}}=I_{m}^{\boldsymbol{k}}$, the $C_{K, m} \times C_{K \cdot m}$ identity matrix.

In order to simplify the hypothesis for the following recurrence formula, we shall extend the definition of $A_{m, n}^{K}$ for all integers $K, m, n$.

Conventions.

(a) Let $\varnothing$ stand for the empty matrix and let $O_{m, n}^{K}$ stand for the $C_{K, m} \times C_{K, n}$ matrix of all zeros. Sometimes $O_{m, n}^{K}$ may be abbreviated by 0 .

(b) $A_{m, n}^{K}=\varnothing$ if $K<m$ or $n$; or if $K, m$ or $n$ is less than zero.

(c) $A_{m, n}^{K}=O_{m, n}^{K}$ if $m<n$.

It is now easy to see that the following formula is true.

LEMMA 3.

$$
A_{m, n}^{K}=\left(\frac{A_{m-1, n-1}^{K-1}}{O_{m, n-1}^{K-1}} \mid \frac{A_{m-1, n}^{K-1}}{A_{m, n}^{K-1}}\right) .
$$

The $A_{m, n}^{K}$ matrices satisfy a multiplication formula which ${ }_{i}^{\pi}$ gives a simple proof of our main result.

Lemma 4 . Let $K \geqq m \geqq p \geqq n \geqq 0$. Then

$$
A_{m, p}^{K} A_{p, n}^{K}=C_{m-n, p-n} A_{m, n}^{K} .
$$

Proof. For $K=0$ or $K=1$ (see Lemma (2a)) the lemma is true.

Now the proof proceeds easily by induction. We multiply $A_{m, p}^{K}$ by $A_{p, n}^{K}$ using the partition formula of Lemma 3 . The resulting matrix is partitioned into four parts. Three parts clearly agree with the stated formula. For the upper right hand submatrix we have

$$
\begin{aligned}
A_{m-1, p-1}^{K-1} A_{p-1, n}^{K-1}+A_{m-1, p}^{K-1} A_{p, n}^{K-1} & =C_{m-1-n, p-1-n} A_{m-1, n}^{K-1}+C_{m-1-n, p-n} A_{m-1, n}^{K-1} \\
& =C_{m-n, p-n} A_{m-1, n}^{K-1}
\end{aligned}
$$

which is the desired submatrix.

4. Before we state our theorems, it is convenient to establish some notation. From now on, $K \geqq m \geqq n \geqq 0$. By $\mathrm{RN}_{m, n}^{K}$, we denote the row null space of $A_{m, n}^{K}$. Similarly, $\mathrm{CN}_{m, n}^{K}$ denotes the column null space of $A_{m, n}^{K}$. If $M^{m}$ and $N^{n}$ are two vector spaces whose vectors are regarded as tuples of numbers, then $M^{m} \oplus N^{n}$ denotes the $m+n$ dimensional vector space given by those $(m+n)$-tuples of numbers such that the first $m$ positions form a vector of $M^{m}$ and the last $n$ positions form a vector of $N^{n}$. 
We define the following $C_{K, m} \times C_{K, m}$ matrix.

$$
T=\left(\begin{array}{c|c}
I_{m-1} & 0 \\
\hline \frac{-1}{m-n} A_{m, m-1}^{K-1} & I_{m}
\end{array}\right)
$$

where $I_{m-1}$ and $I_{m}$ are the $C_{K-1, m-1}$ and $C_{K-1, m}$ dimensional identity matrices respectively. $T$ is a nonsingular matrix. In fact

$$
T^{-1}=\left(\begin{array}{c|c}
I_{m-1} & 0 \\
\hline \frac{1}{m-n} A_{m, m-1}^{K-1} & I_{m}
\end{array}\right)
$$

THEOREM 1. The nonsingular matrix $T$, operating on the right, induces a linear isomorphism from $\mathrm{RN}_{m-1, n}^{K-1} \oplus \mathrm{RN}_{m, n-1}^{K-1}$ to $\mathrm{RN}_{m, n}^{K-1}$.

Proof.

$T A_{m, n}^{K}$

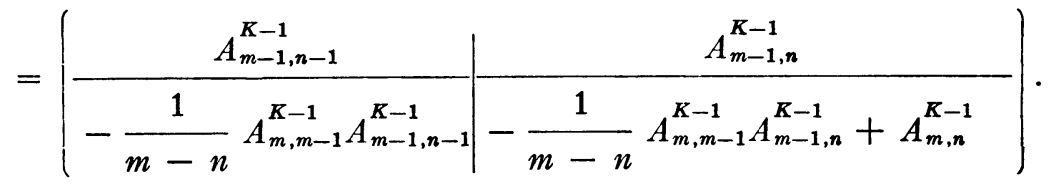

Since $\quad A_{m, m-1}^{K-1} A_{m-1, n-1}^{K-1}=(m-n+1) A_{m, n-1}^{K-1} \quad$ and $\quad A_{m, m-1}^{K-1} A_{m-1, n}^{K-1}=$ $(m-n) A_{m, n}^{K-1}$ (see Lemma 4$)$ we have

$$
T A_{m, n}^{K}=\left(\begin{array}{cc|c}
A_{m-1, n-1}^{K-1} & A_{m-1, n}^{K-1} \\
\hline-\frac{(m-n+1)}{m-n} & A_{m, n-1}^{K-1} & 0
\end{array}\right) .
$$

Since $T$ is nonsingular, right multiplication by $T$ carries the row null space of $T A_{m, n}$ isomorphically to $\mathrm{RN}_{m, n}^{K}$, the row null space of $A_{m, n}^{K}$. Thus all we need do is study the row null space of $T A_{m, n}^{K}$.

Consider the row vector $v=(x, y)$ where $x$ and $y$ are vectors of appropriate length such that

$$
v T A_{m, n}^{K}=\left(x A_{m-1, n-1}^{K-1}-\frac{(m-n+1)}{m-n} y A_{m, n-1}^{K-1}, x A_{m-1, n}^{K-1}\right) .
$$

Then $v T A_{m, n}^{K}=0$ if and only if

$$
x A_{m-1, n-1}^{K-1}-\frac{(m-n+1)}{m-n} y A_{m, n-1}^{K-1}=0
$$


and

$$
x A_{m-1, n}^{K-1}=0 .
$$

The first equation is equivalent to

$$
y A_{m, n-1}^{K-1}=0
$$

since equation (2) and Lemma 4 imply that

$$
x A_{m-1, n-1}^{K-1}=\frac{1}{m-n}\left(x A_{m-1, n}^{\mathbf{K}-1}\right) A_{n, n-1}^{K-1}=0 .
$$

Thus the row null space of $T A_{m, n}^{K}$ is identical with $\mathrm{RN}_{m-1, n}^{K-1} \oplus \mathrm{RN}_{m, n-1}^{K-1}$. This proves the theorem.

Similarly, if we define

we get

$$
L=\left(\begin{array}{c|c}
I_{n-1} & 0 \\
\hline-\frac{1}{m-n} A_{n, n-1}^{K-1} & I_{n}
\end{array}\right)
$$

THEOREM 2. L, operating on the left, induces a linear isomorphism from $\mathrm{CN}_{m, n-1}^{K-1} \oplus \mathrm{CN}_{m-1, n}^{K-1}$ to $\mathrm{CN}_{m, n}^{K}$.

5. In developing the consequences of the above theorems it is helpful to keep the following in mind.

Remark. Suppose $K \geqq m \geqq n \geqq 0$, then $C_{K, m} \geqq C_{K, n}$ if and only if either $m=n$ or $m+n \leqq K$.

Corollary 1.

$$
\begin{aligned}
\operatorname{dim}\left(\mathrm{RN}_{m, n}^{K}\right) & =0 & & \text { if } m+n \geqq K, \\
& =C_{K, m}-C_{K, n} & & \text { if } m+n \leqq K . \\
\operatorname{dim}\left(\mathrm{CN}_{m, n}^{K}\right) & =C_{K, n}-C_{K, m} & & \text { if } m+n \geqq K, \\
& =0 & & \text { if } m+n \leqq K .
\end{aligned}
$$

Proof. The theorem is true for $K=1$ (see Lemma 2). Assume the theorem is true for $K-1$. From the above theorems we see that

$$
\begin{aligned}
& \operatorname{dim}\left(\mathrm{RN}_{m, n}^{K}\right)=\operatorname{dim}\left(\mathrm{RN}_{m-1, n}^{K-1}\right)+\operatorname{dim}\left(\mathrm{RN}_{m, n-1}^{K-1}\right) \\
& \operatorname{dim}\left(\mathrm{CN}_{m, n}^{K}\right)=\operatorname{dim}\left(\mathrm{CN}_{m, n-1}^{K-1}\right)+\operatorname{dim}\left(\mathrm{CN}_{m-1, n}^{K-1}\right) .
\end{aligned}
$$

Suppose $m+n \geqq K$. Then $(m-1)+n \geqq K-1$ and $m+(n-1) \geqq K-1$. So by induction $\operatorname{dim}\left(\mathrm{RN}_{m-1, n}^{K-1}\right)=\operatorname{dim}\left(\mathrm{RN}_{m, n-1}^{K-1}\right)=0$. Hence 
$\operatorname{dim}\left(\mathrm{RN}_{m, n}^{K}\right)=0$. Hence the rank of the matrix must be the number of rows of $A_{m, n}^{K}$, which is $C_{K, m}$ (see Lemma 1). This implies that $\operatorname{dim}\left(\mathrm{CN}_{m, n}^{K}\right)=$ number of columns of $A_{m, n}^{K}$-number of rows $=C_{K, n}$ $-C_{K, m}$. For the other case, when $m+n \leqq K$, use equation (2) and the same argument as above.

Corollary 2. $A_{m, n}^{K}$ has maximal rank.

6. A tactical configuration $C[k, l, \lambda, v],[1]$, is defined as follows. Given a set $E$ of $v$ elements, and given positive in tegers $k, l(l \leqq k \leqq v)$, a tactical configuration is a system of $k$-sets of $E$ such that each $l$-set is contained in exactly $\lambda k$-sets of the system.

CoRollary 3. If $C[k, l, \lambda, v]$ does not contain every $k$-set of $E$, then $k+l<v$.

Proof. A tactical configuration $C[k, l, \lambda, v]$ corresponds to a set of rows, $S$, of $A_{\mathbf{k}, l}^{\mathfrak{r}}$. Let $r_{\boldsymbol{i}}$ be a row vector. Then

$$
\sum_{r_{i} \in S} r_{i}=(\lambda, \lambda, \cdots, \lambda)=\lambda(1, \cdots, 1) .
$$

On the other hand, letting $L=C_{v-l, k-l}$,

$$
\sum_{s 11 r_{i}} r_{i}=L(1, \cdots, 1) \text {. }
$$

Hence we obtain

$$
\sum_{r_{i} \in S} L r_{i}-\sum_{a \| l} \lambda r_{i}=0
$$

or

$$
\sum_{r_{i} \in S}(L-\lambda) r_{i}+\sum_{r_{i} \in S}-\lambda r_{i}=0 .
$$

Since $S$ does not include all the rows of $A_{\mathbf{k}, l}^{v}$, we see that the row vectors form a linearly dependent set. Hence, since $A_{\mathbf{k}, l}^{0}$ has maximal rank, there are more rows than columns. That is, $C_{K, m}>C_{K, n}$, hence $m+n<K$.

\section{BIBLIOGRAPHy}

1. H. Hanani, On some tactical configurations, Canad. J. Math. 15 (1963), 702-722.

2. H. J. Ryser, Combinatorial mathematics, The Math. Assoc. of America, Wiley, New York, 1963.

Institute for Defense Analyses 